Cahiers
de la Recherche
surles Droits Fondamentaux

\section{Cahiers de la recherche sur les droits fondamentaux}

$15 \mid 2017$

Le corps humain saisi par le droit : entre liberté et propriété

\title{
Chronique de jurisprudence du droit des étrangers 2016
}

A Chronicle of the Case Law of the Rights of Aliens 2016

Sarah Benhamouda, Guillaume Dujardin et Grace Gnokam

\section{(2) OpenEdition}

\section{Journals}

Édition électronique

URL : https://journals.openedition.org/crdf/570

DOI : $10.4000 /$ crdf.570

ISSN : 2264-1246

Éditeur

Presses universitaires de Caen

Édition imprimée

Date de publication : 1 novembre 2017

Pagination : 177-188

ISBN : 978-2-84133-858-0

ISSN : 1634-8842

Référence électronique

Sarah Benhamouda, Guillaume Dujardin et Grace Gnokam, « Chronique de jurisprudence du droit des étrangers 2016 ", Cahiers de la recherche sur les droits fondamentaux [En ligne], 15 | 2017, mis en ligne le 01 octobre 2019, consulté le 16 novembre 2022. URL : http://journals.openedition.org/crdf/570 ; DOI : https://doi.org/10.4000/crdf.570 


\title{
Chronique de jurisprudence du droit des étrangers 2016
}

\author{
Sarah BENHAMOUDA \\ Doctorante à l'université de Rouen Normandie \\ Centre universitaire rouennais d'études juridiques (CUREJ, EA 4703)
}

\section{Guillaume DUJARDIN}

Ancien doctorant à l'université de Caen Normandie

Membre associé du Centre de recherche sur les droits fondamentaux et les évolutions du droit (CRDFED, EA 2132)

\section{Grace GNOKAM}

Doctorante à l'université de Rouen Normandie

Centre universitaire rouennais d'études juridiques (CUREJ, EA 4703)

I. Le droit d'asile

A. Un peu de nouveau pour les faits nouveaux...

1. Sur le principe de confidentialité des éléments d'information relatifs à une demande d'asile

2. Sur la reconnaissance du fait nouveau

B. L'unité de famille: quelques ambiguïtés encore

1. Sur la justification de la solution du Conseil d'État: le principe de l'unité de la famille

2. Sur la question des risques de persécution

II. Les libertés des étrangers

A. Prestations familiales et regroupement familial: un mariage de raison?

1. Sur la violation des articles 8 et 14 de la Convention EDH

2. Sur la prise en compte de l'intérêt supérieur de l'enfant (article 3 de la Convention internationale relative aux droits de l'enfant)

B. Le droit au logement comme liberté fondamentale

1. Sur I'article L. 521-3 du Code de justice administrative

2. Une illustration du contrôle normal

\section{Les délais}

A. Une unification des contentieux de l'obligation de quitter le territoire français (00TF)

B. Enfin une responsabilité de l'État pour une durée d'examen d'une demande de titre manifestement excessive

1. L'inapplicabilité logique du $2^{\text {nd }}$ alinéa de l'article R. 121-15 du CESEDA aux ressortissants d'États tiers

2. Une réponse décevante du juge quant à l'action en responsabilité 
Le thème de la migration a encore occupé en 2016 une place prépondérante dans l'actualité; le nombre croissant d'étrangers arrivés sur notre sol n'y étant assurément pas pour rien ${ }^{1}$. Cet engouement médiatique s'est traduit, une fois de plus, par une «grande loi » - du moins d'un point de vue quantitatif $!^{2}-$, celle du 7 mars $2016^{3}$. Parmi moult apports, retenons une énième réforme du contentieux des étrangers ${ }^{4}$, la création des cartes de séjour pluriannuelles ${ }^{5}$ ou bien encore le réaménagement du contrat d'accueil et d'intégration (devenu contrat d'intégration républicaine) ${ }^{6}$.

La jurisprudence en 2016 a été riche en apports pour le droit des étrangers. Il est donc peu de dire que le choix des arrêts chroniqués a laissé place à l'arbitraire. Le choix a toutefois été fait cette année d'ouvrir davantage les présentes colonnes aux décisions des juges du fond. Modelant tout autant notre matière que les «grands arrêts» du Conseil d'État, il a été décidé de donner un peu de visibilité à ces dernières. Certaines jurisprudences, bien qu'importantes, ne seront en outre pas commentées ici, dans la mesure où la doctrine s'y est déjà penchée. Parmi ces «absents de marque», citons l'arrêt Paposhvili c. Belgique qui atténue fortement les effets de la jurisprudence antérieure quant à l'éloignement de personnes atteintes d'une maladie grave ${ }^{7}$; ou bien encore l'arrêt A. M. c. France qui aboutit à une condamnation de la France pour violation de l'article $5, \S 4$ de la Convention européenne des droits de l'homme (Convention EDH), en raison de l'étroitesse du contrôle du juge administratif sur les arrêtés de placement en rétention administrative d'étrangers mineurs ${ }^{8}$.

Comme l'an dernier, les présentes colonnes accorderont une place non négligeable au droit d'asile (I); les droits fondamentaux des étrangers ont connu quelques développements intéressants (II); et la question du délai, tant pour l'examen du titre de séjour que pour le contentieux né de son rejet, doit enfin être abordée (III).

\section{Le droit d'asile}

\section{A. Un peu de nouveau pour les faits nouveaux... ${ }^{9}$}

Notion d'une importance pratique considérable en ce qu'elle détermine en grande partie la recevabilité d'un réexamen de demande d'asile, elle n'a guère intéressé pour le moment la doctrine ${ }^{10}$. L'année 2016 a pourtant donné l'occasion aux juges de l'asile d'y revenir substantiellement. Dès le 7 janvier, la grande formation de la Cour nationale du droit d'asile (CNDA) ${ }^{11}$ a complété le considérant de principe inauguré dans l'arrêt $M^{\text {lle }} \mathrm{Gal}^{12}$. Malgré l'intérêt qu'aurait pu légitimement susciter cet arrêt, il a été fait le choix de jeter un coup de projecteur sur un arrêt du Conseil d'État, rendu le 10 février $2016^{13}$.

Ressortissant sri lankais, M. A. effectue une première demande d'asile, rejetée définitivement par la CNDA le 23 décembre 2011. Le 30 août 2012, le directeur de l'Office français de protection des réfugiés et apatrides (OFPRA) décide de ne pas faire droit à la demande de réexamen; décision confirmée par la CNDA le 5 juin 2013. Entretemps, le 31 janvier 2013, la préfecture de l'Oise sollicite un laissez-passer auprès de l'ambassade du Sri Lanka, en communiquant à celle-ci une copie d'un procès verbal d'audition dressé par la police judiciaire, dans lequel était mentionnée la demande d'asile de l'intéressé.

C'est précisément sur ce dernier élément que se fonde le Conseil d'État pour censurer l'arrêt de la CNDA. Dans un considérant de principe, la haute juridiction affirme:

[...] la confidentialité des éléments d'information relatifs aux personnes sollicitant l'asile en France constitue une garantie essentielle du droit d'asile, lequel est un principe de valeur constitutionnelle; que, par suite, s'il est loisible à l'autorité administrative d'adresser aux autorités du pays d'origine d'un ressortissant étranger en situation irrégulière tout élément en vue de son identification pour

1. Les statistiques de l'immigration en 2016 sont consultables, accompagnées d'un communiqué de presse, à l'adresse: http://www.immigration. interieur.gouv.fr/Info-ressources/Donnees-statistiques/Donnees-de-l-immigration-de-l-asile-et-de-l-acces-a-la-nationalite-francaise.

2. Voir, en ce sens, K. Parrot, "Aperçu critique de la loi du 7 mars 2016 relative au droit des étrangers en France», Recueil Dalloz, 2017, p. 231; M. Bouleau, «La loi n 2016-274 du 7 mars 2016 "relative au droit des étrangers en France” ou les illusions du législateur», Recueil Dalloz, 2016, p. 1720.

3. Loi no 2016-274 du 7 mars 2016 relative au droit des étrangers en France.

4. Voir D. Turpin, «La loi du 7 mars 2016 relative au droit des étrangers en France. Mieux accueillir les uns, mieux éloigner les autres», Revue critique de droit international privé, 2016, p. 235 sq.

5. Art. 17 sq. de la loi du 7 mars 2016, codifié aux art. L. 313-17 sq. du Code de l'entrée et du séjour des étrangers et du droit d'asile (CESEDA).

6. Art. L. 311-9 du CESEDA; voir notre contribution: G. Dujardin, «Les "valeurs républicaines": traduction juridique de la notion de respect?», communication au colloque «Le respect en droit», organisé à Nantes le 3 novembre 2016.

7. Cour EDH, GC, 13 décembre 2016, Paposhvili c. Belgique, $\mathrm{n}^{\circ}$ 41738/10: La semaine juridique, édition générale, $\mathrm{n}^{\circ}$ 52, 2016, 1423, obs. F. Sudre; L'actualité juridique. Droit administratif, 2017, p. 160, chron. L. Burgogue-Larsen; La revue des droits de l'homme, $\mathrm{n}^{\circ}$ 11, 2017, comm. N. Klausser.

8. Cour EDH, 12 juillet 2016, A. M. c. France, $\mathrm{n}^{\circ}$ 56324/13: L'actualité juridique. Droit administratif, 2016, p. 1738, chron. L. Burgogue-Larsen; Lettre "Actualités Droits-Libertés» de la La revue des droits de l'homme, 29 août 2016, comm. A. Gelblat.

9. Par Guillaume Dujardin.

10. Citons tout de même la note de F. Julien-Laferrière sous l'arrêt «fondateur» M"le Gal: L'actualité juridique. Droit administratif, 1995 , p. 399.

11. CNDA, grande formation, 7 janvier $2016, M^{m e} M ., \mathrm{n}^{\circ} 15025487$, cons. 21.

12. CE, sect., 27 janvier 1995, $M^{\text {lle }}$ Gal, $\mathrm{n}^{\circ}$ 129428, JurisData, $\mathrm{n}^{\circ}$ 1995-040410: Droit administratif, 1995, comm. 83, note M. Denis-Linton; L'actualité juridique. Droit administratif, 1995, p. 399, note F. Julien-Laferrière; Revue française de droit administratif, 1996, p. 527, note M. Denis-Linton; La semaine juridique, édition générale, 1995, IV, 840.

13. CE, 10 février 2016, M. A., $\mathrm{n}^{\circ} 373529$. 
assurer la mise en œuvre d'une mesure d'éloignement prise à son encontre, la transmission à ces autorités, après qu'une demande d'asile a été définitivement rejetée, d'informations relatives à l'existence ou au contenu de cette demande constitue un fait nouveau justifiant un nouvel examen de la demande d'asile $[\ldots]^{14}$.

Le raisonnement en deux temps retient l'attention; ces deux phases appelant certaines observations de notre part.

\section{Sur le principe de confidentialité des éléments d'information relatifs à une demande d'asile}

Dans le premier temps de son raisonnement, le Conseil d'État interprète avec une légère souplesse un principe constitutionnel dégagé en 1997 par le Conseil constitutionnel. Saisi de l'article 8-3 de la (future) loi $n^{\text {o }}$ 97-396 du 24 avril 1997, le juge de l'aile Montpensier affirme que «la confidentialité des éléments d'information détenus par [l'OFPRA] relatifs à la personne sollicitant en France la qualité de réfugié est une garantie essentielle du droit d'asile, principe de valeur constitutionnelle ${ }^{15}$. Repris dans une décision de $2003^{16}$, ce principe avait pour objet d'interdire la communication d'éléments relatifs à une demande d'asile à des agents de l'État non habilités à en connaitre. C'est dans cette optique que le Conseil constitutionnel censure une disposition de la loi de 1997 qui permettait à des agents du ministère de l'Intérieur d'accéder à un fichier des empreintes digitales des demandeurs d'asile créé à l'OFPRA ${ }^{17}$.

Dans sa décision de 2003, la juridiction constitutionnelle avait déjà eu l'occasion de circonscrire les conséquences du principe de confidentialité. Elle y avait en effet admis la constitutionnalité d'une disposition qui admettait la communication de «documents d'état civil ou de voyage permettant d'établir la nationalité de la personne dont la demande d'asile est [définitivement] rejetée ${ }^{18}$. C'est visiblement en se fondant sur cette décision que le Conseil d'État admet qu'il

[...] est loisible à l'autorité administrative d'adresser aux autorités du pays d'origine [...] tout élément en vue de son identification pour assurer la mise en ouvre d'une mesure d'éloignement prise à son encontre ${ }^{19}$.

Ce faisant, la juridiction suprême administrative pourrait sembler adopter une position plus permissive que celles du juge constitutionnel dans la mesure où la référence «tout élément en vue de son identification» est logique- ment plus large que celle relative aux «documents d'état civil ou de voyage». Mais il est vrai que la problématique soumise au Conseil d'État est différente de celle soumise au juge constitutionnel. Il est compréhensible que soit admise la communication de documents permettant d'établir la nationalité de la personne aux services de l'État chargés de son éloignement, afin de leur permettre de fixer le pays de destination. Par analogie, il nous semble également possible d'admettre la communication aux autorités consulaires d'éléments permettant d'identifier la personne (la question n'étant plus à ce stade de déterminer sa nationalité).

Suite à cet arrêt, la haute juridiction a, de nouveau, eu l'occasion de se prononcer sur les conséquences pratiques dudit principe de confidentialité. Le circonscrivant encore un peu plus, elle déclarait qu' "aucune règle ni aucun principe ne font obstacle, de manière absolue, à ce qu'il [l'OFPRA] se fonde, pour apprécier le bien-fondé d'une demande d'asile, sur des éléments issus du dossier d'un tiers ${ }^{20}$, alors même que ce tiers s'était opposé à leur communication.

\section{Sur la reconnaissance du fait nouveau}

La position adoptée dans cet arrêt n'est pas inédite. À plusieurs reprises, la CNDA a eu l'occasion de reconnaître l'existence d'un fait nouveau, en cas de transmission aux autorités du pays d'origine du demandeur des éléments relatifs à sa procédure d'asile. Dès 2007, la Commission des recours des réfugiés (CRR) avait estimé que la communication de l'avis de recherche d'un demandeur d'asile mauritanien au consul de Mauritanie

[...] a eu pour effet d'aboutir à son «fichage» par les autorités de Mauritanie qui, ayant non seulement connaissance de l'existence de sa demande d'asile, mais encore du contenu de cette demande qui se présente comme une mise en cause directe desdites autorités, ne peuvent le regarder que comme un opposant politique ${ }^{21}$.

Réitérée à plusieurs reprises par la $\mathrm{CNDA}^{22}$, cette position avait été consacrée en des termes particulièrement nets par le Conseil d'État en 2014:

Considérant que, s'il est loisible à l'autorité administrative d'adresser aux autorités du pays d'origine d'un ressortissant étranger en situation irrégulière tout élément en vue de son identification pour assurer la mise en œuvre d'une mesure d'éloignement prise à son encontre, la transmission à ces autorités, après qu'une demande d'asile a été

14. Ibid., cons. 3 .

15. CC, déc. $\mathrm{n}^{\circ}$ 97-389 DC du 22 avril 1997, cons. 26 : Recueil Dalloz, 1999, p. 237, note F. Mélin-Soucramanien; Revue du droit public, 1997, p. 931, note F. Luchaire; L'actualité juridique. Droit administratif, 1997, p. 524, note F. Julien-Laferrière; Revue française de droit constitutionnel, 1997, p. 571 , note O. Lecucq.

16. CC, déc. $\mathrm{n}^{\circ}$ 2003-485 DC du 4 décembre 2003: Revue du droit public, 2004, p. 565, note F. Luchaire; Revue française de droit constitutionnel, 2004, p. 336, note L. Domingo; Recueil Dalloz, 2004, p. 1279, note O. Le Bot; Recueil Dalloz, 2004, p. 1034, note D. Turpin.

17. CC, déc. $\mathrm{n}^{\circ} 97-389$ DC.

18. CC, déc. $\mathrm{n}^{\circ}$ 2003-485 DC, voir notamment cons. 44

19. CE, 10 février 2016, M. A., cons. 3.

20. CE, 27 juillet $2016, M$. C., $\mathrm{n}^{\circ} 386797$, cons. 4 .

21. CRR, SR, $1^{\text {er }}$ juin $2007, M . O ., \mathrm{n}^{\circ} 05035520 / 561440$

22. CNDA, 29 septembre 2011, M. K., no 10009297; CNDA, 12 mars 2013, M. D., n $\mathrm{n}^{\mathrm{o}} 12012125$ 
définitivement rejetée, d'informations relatives au contenu de cette demande constitue un fait nouveau justifiant un nouvel examen à la demande d'asile $[\ldots]^{23}$.

L'apport de l'arrêt commenté réside donc avant tout dans l'extension du fait nouveau: le fait nouveau ne s'arrête plus à la communication d'éléments relatifs au contenu même de la demande, il s'étend à l'existence de ladite demande. Toutefois, il convient de garder à l'esprit que la reconnaissance d'un fait nouveau ne sera pas systématique dans ces hypothèses: il devra toujours être susceptible de «justifier les craintes de persécution qu'il [le requérant] déclare éprouver ${ }^{24}$. D'ailleurs, dans le considérant 4 de l'arrêt commenté, la haute juridiction prend soin de préciser que la communication à l'ambassade d'éléments relatifs à l'existence de la demande d'asile avait pour effet d'accroître les risques de persécution. De manière générale, il est plausible de penser que cette condition devrait être aisément réunie lorsque l'auteur des persécutions est une autorité étatique; à l'inverse, lorsqu'une autorité non étatique en est à l'origine, la communication d'éléments d'information en violation du principe constitutionnel précité devrait rarement permettre de justifier le réexamen.

Pas complètement inédit donc, l'arrêt ici commenté aura au moins eu le mérite de mettre en lumière l'assouplissement de la condition du fait nouveau, nécessaire pour justifier une demande de réexamen.

\section{B. L'unité de famille: quelques ambiguïtés encore ${ }^{25}$}

Le droit à l'asile est un principe constitutionnellement reconnu et inscrit dans le préambule de la Constitution française. Ce droit assure une protection aux personnes exposées à des risques particuliers dans leur État d'origine et emporte irrémédiablement des conséquences dans la vie des membres de leur famille.

Dans l'arrêt du 11 mai $2016^{26}$, ici chroniqué, le Conseil d'État a dû connaître de deux demandes d'asile présentées par des époux. Il a jugé que la CNDA avait commis une erreur de droit en reconnaissant le statut de réfugié à l'époux et la protection subsidiaire à l'épouse. Les juges du fond ont ainsi ignoré le principe de l'unité de la famille.

\section{Sur la justification de la solution du Conseil d'État: le principe de l'unité de la famille}

Le principe de l'unité de la famille n'est contenu dans aucun instrument législatif ou conventionnel. On peut néanmoins en retrouver une première trace dans l'acte final de la conférence des plénipotentiaires, qui a adopté la Convention de Genève en 1951.

Dès 1957, la CRR a considéré que «la protection que la Convention de Genève a pour but d'assurer au réfugié serait rendue vaine si elle ne s'étendait pas aussi au conjoint $»^{27}$. Par un arrêt de 1994, le Conseil d'État, réuni en assemblée du contentieux, l'a érigé en principe général du droit ${ }^{28}$. Ainsi, l'arrêt du 11 mai 2016 reprend une solution depuis longtemps acquise. Il rappelle et surtout précise les conditions de mise en œuvre du principe de l'unité de la famille.

La première condition est relative à la nationalité. En effet, les époux doivent être ressortissants d'un même État. Cela rendrait vraisemblables les risques similaires invoqués. Ce n'est qu'en l'absence d'une telle protection, assurée par le pays d'origine, que la France peut intervenir. Elle vient donc combler une carence du pays dont le ressortissant a la nationalité ${ }^{29}$. Cette condition peut paraître inadaptée dans le contexte géopolitique mondial actuel. En effet, il ne serait pas surprenant d'être face à des époux ressortissants d'États distincts, ne pouvant, ni l'un ni l'autre, prétendre à une protection de leur État d'origine. Le conjoint du réfugié qui résidait par exemple dans l'État d'origine de celui-ci, et qui ne peut justifier de craintes personnelles, ne se verrait pas appliquer le principe de l'unité de la famille. En l'espèce, la condition de nationalité était bien remplie.

La seconde condition est celle relative à l'existence d'un lien matrimonial. L'arrêt nous signale qu'il faudrait que le demandeur soit "uni par le mariage au réfugié " ${ }^{30}$. Cette règle a été posée, en 1994, à l'occasion de l'affaire Agyepong susmentionnée. Dans cet arrêt, le Conseil d'État a limité le bénéfice du principe aux époux. Dès 1997, il l'a étendu à d'autres hypothèses, à condition toutefois de justifier d' "une liaison suffisamment stable et continue pour former avec lui [le réfugié] une famille $»^{31}$. Il est donc applicable à d'autres formes de conjugalité telles que le concubinage et possiblement le pacte civil de solidarité (PACS). Ces deux formes d'union impliquent une certaine stabilité et une communauté de vie, qui laissent supposer l'existence d'une «famille». Telle est également sa position dans notre arrêt.

Cette dernière condition pourrait, nous semble-t-il, s'appliquer aux époux. Ainsi, en cas de soupçon, la seule preuve du mariage ne suffirait pas à justifier l'application du principe. Il faudrait encore démontrer l'existence d'une vie familiale. Cela permettrait d'éviter par exemple les mariages dits «de complaisance» et donc toute fraude à la

23. CE, 5 novembre $2014, M . A ., \mathrm{n}^{\circ} 369658$, cons. 3 .

24. CE, sect., 27 janvier 1995, $M^{\text {lle }}$ Gal.

25. Par Grace Gnokam.

26. CE, 11 mai 2016, M. et $M^{m e}$ A., $\mathrm{n}^{\circ} 385788$.

27. CRR, 12 mars 1957, Baselga, $\mathrm{n}^{\circ} 1474$.

28. CE, 2 décembre 1994, Agyepong, $\mathrm{n}^{\circ} 112842$

29. CE, 23 février 2009, OFPRA, n 283246 .

30. CE, 11 mai 2016, M. et $M^{m e} A$., cons. 1.

31. Ibid. Avant l'arrêt Agyepong, la CRR avait déjà eu à le faire: voir notamment CRR, 2 juin 1981, Adhihetty, nº 11995. 
loi. Nous pouvons penser que c'est également pour cette même raison, qu'au terme de l'arrêt commenté, ne sont pas pris en compte les mariages célébrés postérieurement à l'introduction de la demande d'asile.

La famille doit être protégée et préservée. Comme a pu le souligner Martine Denis-Linton, dans ses conclusions sous l'affaire Agyepong, l'intérêt d'un tel principe est d'étendre "le champ d'application de la Convention de Genève à la famille proche du réfugié ${ }^{32}$. Dans cette optique, la haute juridiction nous rappelle que cette reconnaissance fait partie du processus de protection qui doit être assurée au réfugié. Ils appliquent donc cette règle avant tout dans l'intérêt du réfugié, «orphelin » d'État ${ }^{33}$, et par ricochet dans celui de son conjoint, comme faisant partie d'un catalogue de garanties qui doivent lui être assurées dans le cadre de la protection dont il bénéficie.

\section{Sur la question des risques de persécution}

Lorsque les conditions en sont réunies, le principe de l'unité de la famille doit être appliqué de plein droit. Les juges ne devraient donc pas rechercher l'existence d'une crainte personnelle du conjoint. Il existerait en quelque sorte une "présomption de [...] persécution ${ }^{34}$ applicable au conjoint. En effet, la vie commune suppose des conditions de vie similaires. Toute situation d'insécurité pesant sur l'un a indéniablement un impact sur l'autre. Selon les termes de Martine Denis-Linton: «[...] la communauté de risques que le mariage ou la filiation fait courir au conjoint et à ses enfants [...] justifie la communauté de statut ${ }^{35}$.

Cependant, lorsque le Conseil d'État indique ici que le statut est reconnu au conjoint du réfugié «à raison des risques de persécutions qu'ils encourent également ${ }^{36}$, nous pouvons nous demander si cela ne constituerait pas un retour en arrière ${ }^{37}$. Cette assertion fait-elle référence à des risques propres ou alors à ceux pesant essentiellement sur l'époux réfugié? Gageons que la seconde interprétation soit la bonne car, sinon, le principe serait vidé de toute son utilité. Son intérêt est bel et bien de permettre au conjoint, qui ne peut justifier de craintes personnelles, de prétendre au statut de réfugié ${ }^{3}$.

Comme les juges du Palais-Royal le rappellent, le principe de l'unité de famille suppose que «la même qualité soit reconnue au conjoint». Ils cassent donc la décision de la CNDA car celle-ci a offert à l'épouse une protection a minima. En effet, les bénéfices de la protection subsidiaire sont beaucoup moins importants que ceux du statut de réfugié. À titre d'exemple, alors que le statut de réfugié confère à son bénéficiaire le droit à une carte de résident d'une durée de dix ans (art. L. 314-11 $8^{\circ}$ du CESEDA), la protection subsidiaire lui confère un titre de séjour mention "vie privée et familiale» d'une durée d'un an (art. L. 712-1 du CESEDA).

En octroyant à l'épouse le bénéfice de la protection subsidiaire, la CNDA reconnaissait l'existence d'un risque qui, s'il ne justifie pas en principe la reconnaissance du statut de réfugié, aurait dû en pareille circonstance (conjoint d'un réfugié) et en tout état de cause, le justifier. La solution du Conseil d'État doit donc être saluée.

Nous pouvons être surpris qu'à ce jour aucune disposition législative n'ait entériné cette règle prétorienne, comme cela est le cas dans d'autres pays européens, à l'instar de l'Allemagne, la Belgique et la Grèce ${ }^{39}$. Relevons néanmoins l'instauration, par la loi no 2015-925 du 29 juillet 2015, d'un corollaire au principe de l'unité de la famille: le droit à réunification familiale (art. L. 752-1 du CESEDA). Ce droit permet au réfugié et au bénéficiaire de la protection subsidiaire d'être rejoint par certains membres de sa famille (le conjoint, le partenaire, le concubin, les enfants... $)^{40}$.

\section{Les libertés des étrangers}

\section{A. Prestations familiales et regroupement familial: un mariage de raison ? ${ }^{41}$}

«Les prestations familiales sont des prestations sociales dont l'objet est d'apporter aux familles une aide compensant partiellement les dépenses engagées pour la subsistance et l'éducation des enfants ${ }^{42}$. En principe, tant les nationaux que les étrangers peuvent en bénéficier, sous réserve de remplir les conditions prévues par la loi.

Par un arrêt du 11 mai $2016^{43}$, le Conseil d'État, saisi d'une demande de regroupement familial, a considéré que «la seule circonstance qu'un refus de regroupement, opposé

32. M. Denis-Linton, «Le maintien de l'unité familiale comme principe général du droit applicable aux réfugiés politiques. Conclusions sur CE, 2 décembre 1994, $M^{\text {me }}$ Agyepong», Revue française de droit administratif, 1995, p. 86.

33. Ibid.

34. L. Touvet, J.-H. Stahl, «Application du principe d'unité de la famille aux réfugiés politiques. Chronique sous CE, ass., 2 décembre 1994 ", L'actualité juridique. Droit administratif, 1994, p. 878.

35. M. Denis-Linton, «Le maintien de l'unité familiale...».

36. CE, 11 mai 2016, M. et $M^{m e} A$., cons. 1 .

37. CE, 25 avril 1990, $M^{\text {me }}$ Francis, $\mathrm{n}^{\circ} 84036$ : « [...] en estimant, après examen particulier du cas de la requérante, lequel devait s'apprécier abstraction faite de la décision susceptible d'intervenir sur la demande de son mari, que celle-ci n'établissait pas la réalité des faits de persécution, la Commission [...] a pu sans commettre d'erreur de droit refuser à la requérante le bénéfice des dispositions [...] de la Convention de Genève».

38. En ce sens, voir M. Denis-Linton, «Le maintien de l'unité familiale...».

39. Voir J. C. Kissangoula, «Le droit des réfugiés: double nationalité et unité familiale. Note sous CE, 23 février 2009, OFPRA», Revue française de droit administratif, 2010, p. 580 .

40. Voir V. Tchen, «Commentaire sous art. L. 752-1», in Code de l'entrée et du séjour des étrangers et du droit d'asile commenté, Paris, LexisNexis, 2017.

41. Par Grace Gnokam.

42. INSEE, «Prestations familiales», 13 octobre 2016, en ligne: https://www.insee.fr/fr/metadonnees/definition/c113o.

43. CE, 11 mai 2016, M. D., nº 392191 . 
en raison de la présence en France de l'enfant, fasse obstacle à la perception des prestations familiales $[. .]$.$» ne suppose$ pas une violation des articles 8 de la Convention EDH et 3 de la Convention internationale relative aux droits de l'enfant.

\section{Sur la violation des articles 8 et 14 de la Convention EDH}

Dans cet arrêt, la demande de regroupement familial est traitée à l'aune de l'une de ses conséquences, en l'occurrence l'octroi ou non de prestations familiales. Ainsi, la Cour effectue une appréciation in concreto eu égard aux circonstances de la cause. En effet, le versement des prestations familiales pour les enfants d'étrangers nés en dehors du sol français est subordonné au respect de la procédure de regroupement familial ${ }^{44}$.

En l'espèce, les deux enfants vivaient depuis plusieurs années en France lorsque la demande de regroupement familiale a été introduite. L'autorité publique pouvait donc valablement rejeter cette demande ${ }^{45}$, en s'assurant néanmoins qu'un tel rejet ne porterait pas une atteinte disproportionnée au droit au respect de la vie privée et familiale et à l'intérêt supérieur de l'enfant ${ }^{46}$. Ladite violation pouvait-elle être caractérisée par l'impossibilité de bénéficier de prestations familiales? De manière contestable, le Conseil d'État y répond par la négative.

Les juridictions judiciaires ont souvent eu à se prononcer sur la question de la conventionnalité de la condition de régularité de l'entrée sur le territoire des enfants nés à l'étranger pour l'octroi de prestations familiales. Notons que de cette conventionnalité peut dépendre la solution du juge administratif, relative au regroupement familial.

Dans un premier temps, la Cour de cassation conclut à l'inconventionnalité de cette condition, au regard des articles sus-évoqués ${ }^{47}$. Dans un second temps, après l'adoption de la loi $\mathrm{n}^{\circ}$ 2005-1579 du 19 décembre $2005^{48}$, elle a infirmé sa position ${ }^{49}$. La Cour européenne des droits de l'homme s'est quant à elle alignée sur cette nouvelle position des juridictions nationales ${ }^{50}$.

La question de l'existence d'une discrimination ne nous semble, pourtant, toujours pas résolue, la réglementation nationale restant parsemée de différences de traitement.

En effet, il existe une différence de traitement évidente entre les nationaux et les personnes de nationalité étrangère. La loi traite également de manière différente les ressortissants d'États membres de l'Union européenne et les ressortissants d'États tiers à l'Union. En effet, la condition de régularité de l'entrée des enfants n'est pas applicable aux premiers (art. L. 512-2, al. $1^{\text {er }}$ du Code de la sécurité sociale). Il s'agit là, il est vrai, de différences de traitement fondées sur une cause objective.

Il existe aussi une différence de traitement entre les parents étrangers d'enfants nés en France et les parents étrangers d'enfants nés à l'étranger. Alors que la seule production de l'acte de naissance de l'enfant dans le premier cas suffit, une condition supplémentaire est recherchée dans le second. Dans le même sens, il existe une différence de traitement entre étrangers, selon le document de séjour qu'ils détiennent ${ }^{51}$. On peut relever à ce niveau qu'une législation allemande, qui instaurait une telle discrimination, a été condamnée par la Cour européenne des droits de l'homme ${ }^{52}$, qui a considéré qu'il n'existait pas de motifs propres à la justifier ${ }^{53}$. Par ailleurs, à la suite de deux arrêts en date du 5 avril 2013, l'assemblée plénière de la Cour de cassation a considéré que cette disposition n'était pas applicable aux ressortissants d'États liés à l'Union européenne par « un accord interdisant toute discrimination fondée sur la nationalité dans le domaine des prestations familiales ${ }^{54}$. C'est le cas notamment de l'Algérie, du Maroc et de la Turquie. Toutes ces dernières différences de traitement ne nous semblent pas objectivement justifiées et nous paraissent disproportionnées au regard du but poursuivi ${ }^{55}$.

44. Art. D. 512-2 du Code de la sécurité sociale (par exemple: production d'un certificat de contrôle médical délivré par l'Office français de l'immigration et de l'intégration).

45. Art. L. 411-6 du CESEDA.

46. CE, 24 mars 2014, Ministère des Affaires sociales, du Travail et de la Solidarité, $\mathrm{n}^{\circ} \mathbf{2 4 9 3 6 9 .}$

47. Cass., Ass. plén., 16 avril 2004, $\mathrm{n}^{\circ}$ 02-30.157: «[...] les étrangers résidant régulièrement en France avec leurs enfants mineurs bénéficient de plein droit des prestations familiales [...]». Voir, dans le même sens, Cass., $2^{\mathrm{e}}$ civ., 6 décembre 2006, nº 05-12.666, Recueil Dalloz, 2007, p. 21.

48. La loi de 2005, dont la constitutionnalité a été reconnue, a modifié l'article L. 512-2 du Code de la sécurité sociale qui a élargi le bénéfice des prestations familiales à d'autres éventualités.

49. Cass., $2^{\mathrm{e}}$ civ., 15 avril 2010, $\mathrm{n}^{\circ}$ 09-12.911: «[...] en statuant ainsi, alors que répondant à l'intérêt de la santé publique et à l'intérêt de la santé de l'enfant, la production du certificat médical exigée à l'appui de la demande de prestations familiales du chef d'un enfant étranger ne porte pas une atteinte disproportionnée au droit à la vie familiale [...]». Voir, dans le même sens, Cass., Ass. plén., 3 juin 2011, $\mathrm{n}^{\circ}$ 09-69.052: ces dispositions "revêtent un caractère objectif justifié par la nécessité dans un État démocratique d'exercer un contrôle des conditions d'accueil des enfants ».

50. Cour EDH, $1^{\mathrm{er}}$ octobre 2015, Selpa Lokongo et autre c. France, $\mathrm{n}^{\mathrm{o}} 7686 \mathrm{o} / 11$, L'actualité juridique. Droit administratif, $2015, \mathrm{p} .1833$.

51. Art. L. 512-2, al. 3 du Code de la sécurité sociale (par exemple: qualité de membre de la famille d'un réfugié ou de bénéficiaire de la protection subsidiaire)

52. Cour EDH, 25 octobre 2005, Niedzwiecki c. Allemagne, no $58-453 / 00$.

53. «[... la Cour ne discerne pas de motifs propres à justifier la différence établie, pour la reconnaissance du droit à percevoir des allocations familiales, entre, d'une part, les étrangers titulaires d'un permis de séjour permanent et, d'autre part, les étrangers non titulaires de pareil permis de séjour et que, partant, il y a eu violation de l'article 14 combiné avec l'article 8 de la CEDH» (G. Azibert, "L'accès aux prestations familiales de l'enfant étranger et la procédure de regroupement familial», Droit social, 2011, p. 813).

54. Cass., Ass. plén., 5 avril 2013, $\mathrm{n}^{\mathrm{o}}$ 11-17.520 et $\mathrm{n}^{\circ}$ 11.18.947. Citation de C. Laurichesse, «La subordination de l'octroi des prestations familiales au respect des règles du regroupement familial ne constitue pas une discrimination fondée sur la nationalité ", Revue critique de droit international privé, 2016, p. 339 sq., $\$ 26$.

55. «Des autorités administratives telles que la HALDE [Haute autorité de lutte contre les discriminations et pour l'égalité] et la Défenseure des enfants (notamment dans un avis du 9 juin 2004) ont estimé que la distinction réalisée pour l'attribution des prestations familiales était contraire au principe de non-discrimination posé par la Convention» (C. Laurichesse, «La subordination de l'octroi des prestations familiales au respect des règles du regroupement familial ne constitue pas une discrimination fondée sur la nationalité», Revue critique de droit international privé, 2016, p. 339). 
L'appréciation de cette décision au regard de l'intérêt supérieur de l'enfant n'est guère plus satisfaisante.

\section{Sur la prise en compte de l'intérêt supérieur de l'enfant (article 3 de la Convention internationale relative aux droits de l'enfant)}

Le Conseil d'État, par sa décision, entend établir l'existence d'un intérêt du mineur au travers de cette disposition. En effet, les juges du Palais-Royal, relèvent

[...] que [...] les dispositions de l'article L. 512-2 du code de la sécurité sociale [ont] pour objectif d'assurer le respect des règles relatives au regroupement familial, dans l'intérêt même de l'enfant pour lequel celui-ci est sollicité $[\ldots]^{56}$.

En d'autres termes, cette disposition vise à assurer des conditions d'accueil optimales pour les mineurs. Le respect de la procédure de regroupement familial garantirait donc une certaine protection du mineur. Le Conseil d'État caractérise ainsi l'intérêt du mineur: telle est l'une des originalités de cette solution. Le Conseil constitutionnel statuant sur la constitutionnalité de la loi de 2005 a dans ce sens relevé la nécessité de vérifier que l'accueillant a la capacité d'offrir à ses enfants « des conditions de vie et de logement décentes ${ }^{57}$ en France.

Il faut dire que pour contrebalancer cette rigueur de la loi, il est possible de bénéficier du regroupement familial à titre de régularisation (art. R. 411-6 du CESEDA) ${ }^{58}$. Il appartient alors aux autorités locales, s'intéressant à la situation personnelle des requérants, de traiter de la demande de regroupement sur place. Les enfants ainsi régularisés doivent pouvoir bénéficier des prestations familiales ${ }^{59}$. C'est au juge administratif de connaître du contentieux relatif à une telle régularisation. D'où l'enjeu de cet arrêt de la haute juridiction administrative.

S'il est évident qu'il y va de l'intérêt de l'enfant qu'il soit accueilli et pris en charge dans de bonnes conditions, nous pourrions nous demander s'il y va nécessairement de son «intérêt supérieur ». L'intérêt supérieur de l'enfant ne se situe-t-il pas plutôt dans la prise en charge de sa santé et de ses conditions de vie? La prestation familiale ne garantit-elle pas une telle prise en charge? La juridiction administrative semble nous donner un élément de réponse lorsqu'elle relève que, dans l'hypothèse où l'état de santé rendrait le versement de cette prestation essentielle, une réponse positive à la demande de regroupement familial aurait pu être de rigueur.

Cette affirmation, pour le moins lapidaire, ne nous satisfait que partiellement. D'une part, elle ne nous dit pas si tel était le cas; d'autre part, ne pouvons-nous pas considérer que la prise en charge de la santé d'un enfant doit toujours être une considération essentielle ${ }^{60}$ ?

Nous ne pouvons conclure ces développements sans relever le traitement particulier qui est réservé au mineur en droit des étrangers. La vulnérabilité du mineur a toujours justifié un traitement plus favorable à son égard. L'article L. 511-4 du CESEDA prévoit dans cette optique que: «Ne peuvent faire l'objet d'une obligation de quitter le territoire français: $1^{\circ}$ L'étranger mineur de dix-huit ans $[\ldots] »$.

Le mineur étranger n'a donc pas à justifier de la régularité de son séjour en France. Or, dans ce cadre, deux régularités sont recherchées: celle de l'entrée et du séjour du parent et celle de l'entrée du mineur. Or cette dernière régularité revient in fine à rechercher la régularité du séjour. Cela nous paraît contraire au droit au respect de sa vie privée et familiale et surtout contraire à son intérêt supérieur.

Le Conseil d'État aurait donc pu considérer que le rejet de la demande de regroupement familial portait atteinte au droit conventionnel.

Nous pourrions peut-être voir, au travers de l'article L. 521-1 du Code de la sécurité sociale, une volonté de contrôler et maîtriser les flux migratoires, en limitant l'immigration irrégulière. L'impossibilité de bénéficier, une fois sur le territoire national, desdites prestations aurait alors un effet dissuasif.

\section{B. Le droit au logement comme liberté fondamentale ${ }^{61}$}

Dans cette espèce, une première ordonnance ${ }^{62}$ imposait aux autorités publiques de prendre les mesures nécessaires pour mettre fin à toutes situations portant atteinte à la dignité humaine. Le juge des référés avait rejeté la demande d'expulsion des occupants sans droits ni titre du jardin des Olieux par la métropole de Lille sur le fondement de l'article L. 521-3 du Code de justice administrative ${ }^{63}$.

56. CE, 11 mai 2016, M. D., cons. 3 .

57. CC, déc. n 2005-528 DC du 15 décembre 2005, cons. 16

58. Circulaire du $1^{\text {er }}$ mars 2000 relative au regroupement familial des étrangers, NOR: MESNoo21265C, abrogée en totalité par celle du 17 janvier 2006, DPM/DMI/2/2005/ et NOR/INT/D/ 06/00009/C.

59. CC, déc. $\mathrm{n}^{\circ} 2005-528$ DC, cons. 18.

60. Art. 24-1 de la Convention internationale relative aux droits de l'enfant: «Les États parties reconnaissent le droit de l'enfant de jouir du meilleur état de santé possible et de bénéficier de services médicaux et de rééducation. [...]». Voir aussi art. 26-1 de cette Convention: «Les États parties reconnaissent à tout enfant le droit de bénéficier de la sécurité sociale, y compris les assurances sociales, et prennent les mesures nécessaires pour assurer la pleine réalisation de ce droit en conformité avec leur législation nationale».

61. Par Sarah Benhamouda.

62. TA Lille, ord., $1^{\text {er }}$ septembre $2016, \mathrm{n}^{\circ} 1607652$.

63. Ibid., cons. 16: "[...] il appartient dès lors à la Métropole européenne de Lille [...] de se rapprocher de l’État, du département du Nord et de la ville de Lille afin de rechercher et de mettre en œuvre, dès que possible et avant l'arrivée du froid, les mesures appropriées pour mettre fin à une situation contraire à la dignité de la personne humaine $[\ldots] »$. 
La trêve hivernale approchant, le requérant saisit une nouvelle fois le juge administratif et demande au maire de Lille ainsi qu'au préfet du Nord de procéder à la réquisition d'un ou plusieurs bâtiments sur la commune de Lille permettant ainsi qu'un accompagnement pour leurs besoins spécifiques. Par une ordonnance du 20 octobre $2016^{64}$, le juge estime qu'il appartient aux autorités de l'État ${ }^{65}$ de mettre en place toutes les mesures nécessaires pour les personnes en détresse et pour les demandeurs d'asile ainsi que pour les mineurs et les mineurs isolés au risque de porter une atteinte grave à une liberté fondamentale ${ }^{66}$. En plein cœur de l'actualité, les camps de migrants sont à déplorer. En effet, la plupart du temps, il est difficile pour les étrangers d'y résider longuement tant les conditions de vie sont difficiles. Cette difficulté est d'autant plus sérieuse lorsque des jeunes étrangers ou des mineurs isolés sont en cause. Cette catégorie d'étranger vulnérable ${ }^{67}$ a besoin de conditions particulières pour pouvoir vivre. Face à ces situations, les autorités publiques disposent de pouvoirs et doivent éviter une quelconque atteinte aux libertés fondamentales. Ainsi, le droit à l'hébergement d'urgence est considéré comme une liberté publique pouvant être invoquée devant le juge des référés. Le juge administratif appréciera rigoureusement la légalité des mesures prises par les autorités administratives compétentes par la théorie du bilan coût/ avantage.

\section{Sur l'article L. 521-3 du Code de justice administrative}

Cet article dispose qu'

En cas d'urgence et sur simple requête qui sera recevable même en l'absence de décision administrative préalable, le juge des référés peut ordonner toutes autres mesures utiles sans faire obstacle à l'exécution d'aucune décision administrative.

Découlent de cette disposition trois conditions à remplir pour répondre à la recevabilité de ce référé conservatoire (ou mesures utiles). Tout d'abord, doit être démontrée une urgence. Ensuite, il faut démontrer que la mesure demandée est utile. Enfin, cette mesure de doit pas faire obstacle à l'exécution d'aucune décision administrative.

En l'espèce, les requérants invoquent que l'urgence est caractérisée par l'approche de la trêve hivernale et estiment que la défaillance des autorités publiques conduit à une atteinte à une liberté fondamentale et à une atteinte à la dignité humaine. C'est ce qui suscite notre attention puisque cette atteinte porterait sur le droit à l'hébergement et sur la situation des mineurs étrangers.

En présence de mineurs isolés, nul ne peut douter de leur intérêt à agir. Toutefois, étant mineurs et isolés peuvent-ils justifier d'une capacité à agir? Le Conseil d'État a déjà admis qu'un mineur isolé pouvait invoquer un droit à l'hébergement d'urgence dans le cadre du référéliberté «lorsqu'il sollicite un hébergement d'urgence qui lui est refusé par le département auquel le juge judiciaire l'a confié ${ }^{68}$. Cette hypothèse n'est qu'à moitié justifiée ici puisque le département du Nord ne faillit pas à sa compétence. Dans ce cas, le juge des référés est saisi sur le fondement de l'article L. 521-3 du Code de justice administrative. Cette condition de la capacité semblerait ici remplie car le juge apprécie positivement le recours.

On sait en effet que, depuis une décision du 10 février $2012^{69}$, le Conseil d'État estime que le droit à un hébergement d'urgence peut être invoqué dans le cadre du référéliberté $^{70}$. Le Palais-Royal crée dans cette ordonnance une nouvelle liberté fondamentale à classer dans la catégorie des droits-créances ${ }^{71}$. Ce droit à l'hébergement d'urgence est issu d'une loi du 25 mars $2009^{72}$ et permet aux sansabri d'invoquer un droit à l'hébergement d'urgence et de bénéficier d'un accompagnement personnalisé jusqu'à ce qu'une solution adéquate soit trouvée ${ }^{73}$. Ainsi, l'administration est tenue de prendre des mesures, répondant certes à la protection de l'ordre public, mais ne portant atteinte à aucune liberté fondamentale ${ }^{74}$.

En l'espèce, les requérants invoquent une atteinte à une liberté fondamentale et à la dignité humaine lorsque le préfet ne satisfait pas complètement à l'hébergement d'urgence des étrangers. La responsabilité de l'État serait

64. TA Lille, ord., 20 octobre 2016, M. Kanfory C., $\mathrm{n}^{\circ} 1607652$.

65. Sur le fondement de l'article L. 521-3 du Code de justice administrative et de l'article L. 345-2 sq. du Code de l'action sociale et des familles.

66. TA Lille, ord., 20 octobre 2016, M. Kanfory C., cons. 7: cette obligation doit d'autant plus être respectée dès lors qu'un «mineur privé de la protection de sa famille est sans abri et que sa santé, sa sécurité ou sa moralité est en danger [au risque de porter] atteinte [...] à une liberté fondamentale».

67. Voir C. Neirinck, «L'@MIE des enfants isolés étrangers », Droit de la famille, nº 11, 2013, repère 10 : il s'agit ici de l'arrêté du 6 septembre 2013 par lequel le ministère de la Justice invitait à accorder une protection à ces mineurs qui sont des enfants en danger, particulièrement vulnérables en raison de leur isolement, de leur qualité d'étrangers et de leurs parcours.

68. L. Domingo, "Le refus de prise en charge des mineurs isolés étrangers par les départements", La semaine juridique, édition administrations et collectivités territoriales, $\mathrm{n}^{\circ} 4,2015,2019$.

69. CE, réf., 10 février 2012, Fofana, no 356456.

70. Art. L. 521-2 du Code de justice administrative.

71. Voir Olivier Le Bot, «Le droit à un hébergement d'urgence invocable en référé-liberté», La semaine juridique, édition administrations et collectivités territoriales, $\mathrm{n}^{\circ} 7,2012,2059$.

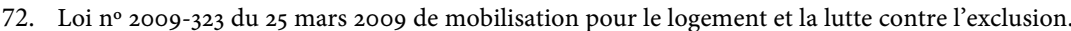

73. Art. L. 352-2-2 du Code de l'action sociale et des familles.

74. Voir C. Boyer-Capelle, «Le juge administratif et les conditions matérielles d'accueil des demandeurs d'asile. Entre protection et pragmatisme», La semaine juridique, édition générale, $\mathrm{n}^{\circ} 17,2013$, doctrine 483 , point $\mathrm{n}^{\circ} 4$ : à ce titre on peut relever : «Le juge administratif porte depuis longtemps une attention particulière aux modalités générales de mise en œuvre du dispositif. Conscient de la situation de faiblesse économique et sociale du demandeur d'asile, il a progressivement renforcé la justiciabilité du droit à des conditions matérielles d'accueil décentes et n'hésite pas à rappeler à l'ordre des pouvoirs publics tentés d'adopter une lecture restrictive des garanties générales offertes ». 
ainsi engagée lorsque, par inaction, une atteinte serait portée à une liberté fondamentale ${ }^{75}$.

\section{Une illustration du contrôle normal}

Le juge contrôle concrètement le projet des autorités afin de déterminer si leur action ne bafoue pas le droit à l'hébergement des requérants. Il déduit ainsi qu'il est plus simple à mettre en œuvre que la réquisition des logements demandée par les requérants. Les autorités bénéficiaient d'une certaine marge d'appréciation et le juge vérifie si une erreur d'appréciation n'a pas été commise ${ }^{76}$. Par ailleurs, relativement aux mineurs isolés, le juge des référés fait aussi une appréciation concrète et estime alors que le plan d'action mis en place par les autorités permet aux mineurs isolés et aux jeunes étrangers de conserver leurs liens avec la commune de Lille en mettant à leur disposition des transports. Cette catégorie peut ici être considérée comme vulnérable ${ }^{77}$ en ce que les mineurs isolés étrangers ont des besoins spécifiques. Ils relèvent de la compétence du département comme en dispose l'article L. 112-3 du Code de l'action sociale et des familles ainsi que le Conseil d'État dans une ordonnance du 10 mars $2014^{78}$.

Le juge des référés conclut ainsi au coût élevé de la réquisition et à la longueur et la lourdeur de la procédure qui dépasseraient la trêve hivernale.

\section{Les délais}

\section{A. Une unification des contentieux de l'obligation de quitter le territoire français $(00 T F)^{79}$}

Le requérant est un ressortissant marocain entré en France le 25 septembre 2012 grâce à un visa de type $C$ délivré par les autorités consulaires espagnoles valable pour une entrée et un séjour de trente jours sur le territoire des États Schengen. Interpellé le 25 octobre 2015, la préfète de la Somme a pris à son encontre une OQTF sur le fondement du $2^{\circ} \mathrm{du}$ I de l'article L. 511-1 du CESEDA. Cette OQTF n'a pas de délai et désigne le Maroc comme pays de renvoi. Par le même arrêté, la préfète a refusé le séjour du requérant sans que le requérant n'ait formulé de demande.
Il saisit le tribunal administratif d'Amiens et demande l'annulation de l'arrêté du 22 octobre 2015. À l'appui de sa demande, il invoque que l'arrêté attaqué a été signé par une autorité incompétente faute d'avoir reçu, à cet effet, une délégation de signature régulière. Il ajoute que l'arrêté attaqué est insuffisamment motivé, est entaché d'erreur de droit, méconnaît les dispositions de l'article L. 313-14 du CESEDA, porte une atteinte disproportionnée à son droit au respect de sa vie privée et familiale en méconnaissance de l'article 8 de la Convention EDH. Il soutient également que l'OQTF est illégale du fait de l'illégalité de la décision de refus de séjour. Par ailleurs, il conteste la décision fixant le pays de renvoi, qui méconnaît les stipulations de l'article 3 de la Convention EDH.

Sur la question de l'annulation de l'OQTF sans délai avec pays de renvoi, le tribunal administratif d'Amiens ${ }^{80}$ rejette la requête au motif que le délai de recours de quarante-huit heures n'a pas été respecté.

Sur le refus de délivrer un titre de séjour, le tribunal administratif a dû répondre à une question nouvelle. Il l'a alors transmise au Conseil d'État en vertu de l'article L. 113-1 du Code de la justice administrative.

La question est la suivante:

Considérant que lorsque, par le même arrêté, le préfet d'une part, refuse de délivrer un titre de séjour à un étranger qui se maintient sur le territoire français au-delà du délai de validité de son visa et, d'autre part, l'oblige à quitter sans délai le territoire français après avoir constaté que celui-ci se trouve dans le cas prévu au $2^{\circ}$ du I de l'article L. 511-1 du code de l'entrée et du séjour des étrangers et du droit d'asile, et qu'il existe un risque qu'il se soustraie à une mesure d'éloignement, au sens du b) du $3^{\circ}$ du II du même article, le délai de recours de quarante-huit heures prévu au II de l'article L. 512-1 du même code est-il applicable aux conclusions dirigées par l'intéressé contre la décision de refus de titre de séjour, alors même que cette décision n'est pas assortie d'une obligation de quitter le territoire français dans les conditions prévues par le $3^{\circ}$ du I de l'article L. 511-1 cité au point 2 et que la légalité de l'obligation prise à son encontre ne dépend pas de celle de la décision relative au séjour?

Sur les fondements des articles L. 511-1 et L. 512-1 du CESEDA, le Conseil d'État ${ }^{81}$ estime que, d'une part, l'étranger faisant l'objet d'une OQTF sans délai dispose

75. On constate toutefois que les cas de voies de fait tendent à diminuer depuis quelques temps et que le juge administratif voit sa compétence élargie dans l'appréciation de la responsabilité de l'administration. En ce sens, voir TC, 17 juin 2013, M. Bergoend c. Société ERDF Annecy Léman, $\mathrm{n}^{\circ} 3911$ et, sur la restriction de la voie de fait, S. Gilbert, «Restriction de la voie de fait», Droit administratif, nº 12, 2013, comm. 86.

76. TA Lille, ord., 20 octobre 2016, M. Kanfory C., cons. 12: «[...] la réquisition de logements d'habitation vacants demandée par les requérants [...] implique le respect d'une procédure lourde comportant plusieurs étapes [...], la réponse du propriétaire qui dispose de deux mois pour dire s'il met fin à la vacance ou s'il s'engage à effectuer les travaux nécessaires à la location et enfin la décision du préfet d'arrêter la procédure ou de procéder à la réquisition; [...] il est impossible de mener à terme une telle procédure avant le $1^{\text {er }}$ novembre, date à laquelle débute la période de la trêve hivernale».

77. CJUE, 27 février 2014, C-79/13, $\$ 9$ : «Dans la législation nationale transposant les dispositions du chapitre II relatives aux conditions matérielles d'accueil et aux soins de santé, les États membres tiennent compte de la situation particulière des personnes vulnérables, telles que les mineurs, les mineurs non accompagnés, les handicapés, les personnes âgées, les femmes enceintes, les parents isolés accompagnés de mineurs et les personnes qui ont subi des tortures, des viols ou d'autres formes graves de violence psychologique, physique ou sexuelle».

78. CE, réf., 10 mars 2014, Département de l'Aveyron et autres, $\mathrm{n}^{\circ} 375279$.

79. Par Sarah Benhamouda.

80. TA Amiens, $3^{\mathrm{e}}$ ch., 23 février 2016, $\mathrm{n}^{\circ} 1503147$.

81. CE, avis, 22 juillet 2016, $\mathrm{n}^{\circ} 398374$, publié aux tables du Recueil Lebon. 
de quarante-huit heures pour pouvoir la contester ainsi que les décisions relatives au séjour. Le requérant pourra «jusqu'à la clôture de l'instruction, former des conclusions dirigées contre toute autre de ces décisions ${ }^{{ }^{8}}$.

D'autre part, il estime que le délai de recours de quarante-huit heures est également applicable, dans le cas où l'étranger se trouverait dans la situation prévue au $2^{\circ}$ du I de l'article L. 511-1 du CESEDA ${ }^{83}$ alors qu'il n'a pas saisi l'administration d'une demande de titre de séjour,

[...] aux conclusions dirigées par le requérant contre le refus de séjour, qui, ayant été notifié en même temps que l'obligation de quitter le territoire sans délai, est indissociable et suit le même régime contentieux $[\ldots]^{84}$.

Le Conseil d'État dégage ici deux configurations où le régime contentieux du titre de séjour suit le régime contentieux de l'OQTF ${ }^{85}$. Le délai de recours est de quarante-huit heures pour toutes les décisions prises en même temps que l'OQTF même pour le titre de séjour pris par une autorité administrative sans même que l'étranger en ait fait la demande.

C'est en ce sens que l'avis du Conseil d'État constitue un éclairage considérable: les contentieux étant alignés, la procédure des délais de recours n'est guère problématique. On peut d'ailleurs soulever que cet alignement répondrait à l'esprit du législateur de $2006^{86}$. Il s'agirait de réunir toutes les mesures prises dans le cadre de l'OQTF de sorte à les traiter dans un même contentieux. L'objectif, connu de tous en contentieux des étrangers, consiste à désengorger les tribunaux ${ }^{87}$ et à faciliter le contentieux ${ }^{88}$. Toutefois, on peut se demander si, par le refus du titre de séjour et lorsque le délai de recours de quarante-huit heures pour contester une OQTF est dépassé, l'étranger ne se trouve pas dans une situation délicate d'autant qu'il invoque le respect de l'article 8 de la Convention EDH. Si l'OQTF ne peut plus être contestée, elle devient exécutoire. Si l'étranger ne dispose pas de titre de séjour, il ne peut rester sur le territoire. C'est d'ailleurs cette question de refus de titre de séjour spontané ${ }^{89}$ qui est intéressante à étudier en l'espèce puisque, d'une part, l'administration prend une mesure sans même que l'étranger en fasse la demande et d'autre part, il ne constitue pas le fondement de l'OQTF.
Le CESEDA prévoit que le préfet peut prendre un refus de titre de séjour à la suite d'une demande d'un étranger ${ }^{\circ}$. Face à ce refus, le CESEDA offre un régime contentieux correspondant à la contestation classique d'une décision administrative. Il s'agit du délai de deux mois à respecter dans le cadre d'un recours en annulation. Ainsi, l'étranger peut bénéficier des garanties offertes contre un refus de titre de séjour. En vertu de la loi du 11 juillet 1979, repose sur l'administration l'obligation de motivation de l'acte pris qu'il soit implicite ou explicite avec la précision suivante: si l'acte est implicite, l'intéressé peut, dans un délai d'un mois, demander la motivation au préfet. L'étranger pourra également saisir une commission départementale de titre de séjour qui doit être saisie par le préfet pour avis lorsqu'il souhaite prendre un refus de titre de séjour ${ }^{91}$. Le non-respect de cette procédure peut «vicier le refus de titre de séjour qui est opposé in fine à l'étranger ${ }^{92}$.

Or, en l'espèce, le refus de titre de séjour a été pris par le préfet, à la suite d'une OQTF, sans même que le requérant en fasse la demande et il ne constitue pas le fondement de l'OQTF. Ce qui a en effet motivé le préfet est l'expiration du visa de l'intéressé. L'étranger ne s'est alors pas présenté au guichet. Il a déjà été jugé que le préfet pouvait prendre une décision de refus de titre de séjour spontanée lorsque le titre de séjour constitue le fondement de l'OQTF. Le tribunal administratif de Toulouse a ainsi jugé légal «le refus de délivrance ou de renouvellement de titre de séjour sans commettre de détournement de procédure ni d'erreur de droit ${ }^{93}$. Le juge toulousain avait fondé sa décision sur une combinaison des articles L. 511-1 et L. 512-1 du CESEDA. Même si les faits commentés et les faits de cette affaire toulousaine sont distincts, dans les deux cas les deux articles constituent le visa des juges.

Ce qui pourrait susciter notre interrogation, c'est l'article 8 de la Convention EDH qui est soulevé par le requérant et qui semblerait avoir subi une double atteinte tant par le refus de séjour que par l'OQTF. Relativement au refus de titre de séjour pris sans demande, l'étranger n'ayant pas déposé de dossier de titre de séjour, sa situation familiale n'a pu être appréciée par le préfet $^{94}$. On peut d'ailleurs se demander si ce refus de séjour spontané ne

82. CE, avis, 22 juillet 2016, pt 4 .

83. Le $2^{\circ}$ du I de l'article L. 511-1 du CESEDA prévoit que: «L'autorité administrative peut obliger à quitter le territoire français un étranger non ressortissant d'un État membre de l'Union européenne, d'un autre État partie à l'accord sur l'Espace économique européen ou de la Confédération suisse et qui n'est pas membre de la famille d'un tel ressortissant au sens des $4^{\circ}$ et $5^{\circ}$ de l'article L. 121-1, lorsqu'il se trouve dans l'un des cas suivants : [...] $2^{\circ} \mathrm{Si}$ l'étranger s'est maintenu sur le territoire français au-delà de la durée de validité de son visa ou, s'il n'est pas soumis à l'obligation de visa, à l'expiration d'un délai de trois mois à compter de son entrée sur le territoire sans être titulaire d'un premier titre de séjour régulièrement délivré».

84. CE, avis, 22 juillet 2016, pt 5 .

85. Voir L. Erstein, "OQTF sans délai, le recours de 48 heures pour tout», La semaine juridique, édition générale, $\mathrm{n}^{\circ}$ 34, 2016 , act. 677.

86. Loi $\mathrm{n}^{\circ}$ 2006-911 du 24 juillet 2006 relative à l'immigration.

87. Voir F.-N. Buffet, Rapport fait au nom de la commission des lois, de la législation, du suffrage universel, du Règlement et d'administration générale sur le projet de loi relatif à l'immigration et à l'intégration, n³ 371 (2005-2006), Sénat, déposé le 31 mai 2006.

88. Voir CNCDH, Avis sur la réforme du droit des étrangers, 21 mai 2015.

89. Voir L. Gros, «Peut-il exister un refus de séjour spontané?», L'actualité juridique. Droit administratif, 2008, p. 590.

90. Art. L. 311-1 du CESEDA.

91. Art. L. 312-1 et L. 312-2 du CESEDA.

92. V. Tchen, Droit des étrangers, $2^{\mathrm{e}}$ éd., Paris, Ellipses, 2011, p. 65.

93. TA Toulouse, 18 septembre $2007, \mathrm{n}^{\circ} 072810$.

94. CAA Paris, 20 juin 2005, nº $04 \mathrm{PAO}_{3625 .}$ 
pourrait pas constituer un vice de compétence. Le contrôle de la légalité externe de ce refus échappe à l'œil du juge administratif ${ }^{95}$ puisque le délai de recours a été dépassé. Relativement à l'OQTF, le délai de recours étant épuisé, le juge ne pourra apprécier l'erreur de droit et vérifier si l'étranger ne bénéficie pas d'une protection empêchant son éloignement ${ }^{96}$.

Dans cette affaire, le requérant n'a pas saisi le juge dans le délai de quarante-huit heures, la mesure d'OQTF étant exécutoire, il doit alors quitter le territoire. La possibilité du référé-liberté lui reste ouverte ${ }^{97}$.

\section{B. Enfin une responsabilité de l'État pour une durée d'examen d'une demande de titre manifestement excessive ${ }^{98}$}

Engorgées de demandes de titres de séjour, ou faisant preuve parfois d'une bonne volonté qui prête à caution, les préfectures les examinent dans des délais souvent particulièrement longs, plaçant de ce fait les usagers dans une grande situation de précarité. Conformément aux indications données par les circulaires, le premier récépissé est en principe d'une durée de validité de quatre mois ${ }^{99}$, et peut être renouvelé une fois ${ }^{100}$.

Devant ces pratiques "plurielles », et qui nuisent largement aux publics concernés, ne serait-il pas temps de permettre aux étrangers d'obtenir réparation des préjudices qui leurs sont causés par elles? Le jugement du tribunal administratif d'Amiens en date du 16 septembre $2016^{101} \mathrm{y}$ apporte quelques éléments de réponse. Le requérant demandait en l'espèce réparation des préjudices causés par la lenteur de l'administration pour répondre à sa demande de régularisation (présentée sur le fondement de l'article L. 313-14 du CESEDA), plus précisément des salaires qu'il n'a pu percevoir, alors qu'il présentait une promesse d'embauche. Pour rejeter l'action en responsabilité, le tribunal commence par réaffirmer que l'article R. 121-15 du CESEDA ne s'applique pas aux ressortissants d'États tiers; puis, estimant que le requérant ne remplissait pas les conditions pour se voir délivrer la carte de séjour prévue à l'article L. 313-14 du CESEDA, le préfet n'a pas commis d'illégalité fautive. Les deux temps du raisonnement appelleront quelques observations de notre part.

\section{L'inapplicabilité logique du $2^{\text {nd }}$ alinéa de l'article R. 121-15 du CESEDA aux ressortissants d'États tiers}

Le $2^{\text {nd }}$ alinéa de l'article R. 121-15 dispose que «[1]a délivrance de la carte de séjour aux ressortissants d'un État tiers intervient au plus tard dans les six mois suivant le dépôt de la demande». Inséré dans le titre II du Code, consacré aux citoyens de l'Union européenne, aux ressortissants des États parties à l'accord sur l'Espace économique européen et aux ressortissants de la confédération Suisse, le juge amiénois considère qu'il n'est pas applicable, contrairement à ce que pourrait laisser penser la formulation passablement ambiguë de cet article, «aux ressortissants d'un État tiers». Dès lors, en ne respectant pas le délai de six mois prévu à cet article, le préfet n'a pas commis d'illégalité fautive ${ }^{102}$.

Cette position confirme les positions de certaines cours administratives d'appel. La cour administrative d'appel de Versailles avait ainsi estimé qu'une ressortissante chinoise ne pouvait se prévaloir de cet article pour faire annuler l'OQTF qui la visait ${ }^{103}$. De manière plus lapidaire - et plus ambiguë - la cour administrative d'appel de Marseille ${ }^{104}$ se contentait d'affirmer que «les dispositions de l'article R. 121-15 [...] ne peuvent être utilement invoquées pour contester la légalité d'un refus de titre de séjour ${ }^{105}$.

\section{Une réponse décevante du juge quant à l'action en responsabilité}

La responsabilité de l'État ne pouvant être recherchée sur la base de la violation de l'article R. 121-15 du CESEDA, le requérant a plaidé une rupture d'égalité devant les charges publiques, à raison du caractère déraisonnable de la durée d'examen du titre de séjour. En effet, entre septembre 2009 et décembre 2013, il ne lui a été délivré que des autorisations provisoires de séjour ne l'autorisant pas à travailler. Pour rejeter l'action, le juge se contente d'indiquer que l'intéressé ne pouvait légalement prétendre à la délivrance de la carte de séjour mentionnée à l'article L. 313-14; et que, par suite, en s'abstenant de lui délivrer ledit titre de séjour, le préfet n'a commis aucune illégalité de nature à engager la responsabilité de l'État. En conséquence, «quel que soit le délai dans lequel l'administration a instruit la demande

95. CE, Ass., 19 avril 1991, Belgacem et CE, Ass., 19 avril 1991, Babas, ${ }^{\circ} 107470$.

96. Voir F. Jault-Seseke, S. Corneloup, S. Barbou des Places, Droit de la nationalité et des étrangers, Paris, PUF (Thémis. Droit), $2015, \mathrm{p} .568$.

97. Art. L. 521-2 du Code de justice administrative.

98. Par Guillaume Dujardin.

99. Le CESEDA est, il est vrai, assez souple en la matière puisqu'il ne fait qu'indiquer que le récépissé délivré ne peut être d'une durée de validité inférieure à un mois, et peut être renouvelé (art. R. 311-5).

100. Circulaire du 5 janvier 2012, NOR: IOC/L/12/o0311/C, pt 1.1: «En tout état de cause, la délivrance de plus de deux récépissés pour un même titre de séjour, en première demande comme en renouvellement, ne devrait rester que très exceptionnelle».

101. TA Amiens, $1^{\text {re }}$ ch., 16 septembre 2016, Thierry Z., $\mathrm{n}^{\mathrm{o}} 1600543$

102. TA Amiens, $1^{\text {re }}$ ch., 16 septembre 2016, Thierry Z., cons. 2.

103. CAA Versailles, 13 octobre $2015, M^{m e} A$., $\mathrm{n}^{\circ} 15$ VEoo729.

104. CAA Marseille, 4 juillet 2016, M. B., $\mathrm{n}^{\circ}$ 15MA05032.

105. Le moyen est-il écarté parce qu'inopérant pour faire annuler un refus de titre, ou à raison de l'inapplicabilité dudit article aux ressortissants marocains? 
de M. Z., ce dernier ne saurait rechercher la responsabilité de l'État en réparation des pertes de revenus ${ }^{106}$.

Cette position du juge amiénois est assez lacunaire, et laisse sans réponse un certain nombre de questions. La première - serait-on tenté de dire la principale - est celle de savoir si un délai manifestement excessif pour prendre la décision engage la responsabilité de l'État, soit sur le terrain de la faute, soit sur celui de l'inégalité devant les charges publiques. Au vu de la jurisprudence antérieure, on serait pourtant enclin à répondre au premier versant de la question par l'affirmative. Une cour administrative d'appel a ainsi estimé que la délivrance tardive d'un récépissé de demande de carte de séjour est fautive, dès lors que le dossier de l'intéressé est complet ${ }^{107}$. Il en va de même pour l'exécution tardive d'une décision de justice enjoignant au préfet de délivrer une carte de séjour, sauf changement dans les conditions de droit ou de fait ${ }^{108}$. De façon encore plus explicite, certaines cours administratives d'appel ont recherché si la difficulté du dossier justifiait le délai de réponse anormalement long. Il a par exemple été jugé que

[...] le délai de vingt mois qui s'est écoulé entre l'annulation du refus implicite de titre de séjour prononcée par le tribunal administratif de Nice pour un motif de légalité externe et l'obtention de son titre de séjour n'est pas anormal, dans les circonstances de l'espèce, le préfet ayant fait valoir, sans être sérieusement contesté par l'intéressé, que le traitement de son dossier avait posé des difficultés particulières ${ }^{109}$.

Et, dans une affaire très proche de la nôtre, une cour administrative d'appel, après avoir constaté que la requé- rante ne remplissait pas les conditions pour obtenir le titre de séjour convoité, a indiqué que le délai de seize mois pendant lequel l'administration a instruit la demande n'était pas excessif dans les circonstances de l'espèce ${ }^{110}$.

La seconde question est relative à l'espèce: l'absence de réparation n'est-elle pas en réalité due à un défaut de lien de causalité? Au regard des faits de l'espèce, nous sommes enclin à le penser. Le requérant demandait ici réparation des salaires qu'il n'a pu percevoir entre 2009 et 2013, son autorisation provisoire de séjour ne lui donnant pas le droit de travailler. Or, il est probable que le juge ait ici considéré que cette absence de salaire ne trouvait pas sa cause dans la longue durée d'examen de la demande de titre mais dans l'absence de sa délivrance (qui était légale - voir supra). En effet, le refus implicite de délivrance de la carte de séjour étant justifié, l'intéressé n'avait pas le droit de travailler au cours de cette période ${ }^{111}$. En revanche, si un préjudice moral avait été invoqué (notamment du fait de la précarité de sa situation), le lien de causalité entre le fait générateur de responsabilité et le préjudice aurait existé nous semble-t-il.

Implicitement donc, il ne semble pas que ce soit l'existence même de cette responsabilité qui soit remise en cause en l'espèce, mais bien les circonstances particulières de l'espèce qui l'ont empêché d'aboutir. Heureusement, serait-on tenté de dire... Les circulaires du ministère de l'Intérieur ne parvenant pas à mettre un terme au phénomène dénoncé plus haut, il serait de bon ton que les juges s'y attaquent sérieusement, certes par des moyens détournés.

106. TA Amiens, $1^{\text {re }}$ ch., 16 septembre 2016, Thierry Z., cons. 6.

107. CAA Bordeaux, 30 juin 2011, Alexandro A., n ${ }^{\circ}$ 10BXo1417.

108. CAA Paris, 18 septembre 2014, Dimitri A., $\mathrm{n}^{\circ}{ }_{13}$ PAoo 745.

109. CAA Marseille, $1^{\text {re }}$ ch., 26 janvier 2015, M. D., n $\mathrm{n}^{\circ}$ 12MAo4838. En l'espèce, le PACS conclu par le requérant était suspecté être de complaisance par le préfet des Alpes-Maritimes; des enquêtes de communauté de vie ayant d'ailleurs été diligentées les 6 février 2009,23 novembre 2010 , 11 février 2011 et 15 avril 2011.

110. CAA Nancy, $2^{\mathrm{e}}$ ch., 12 mai 2016, $M^{\mathrm{ll}} A$., $\mathrm{n}^{\mathrm{o}} 15 \mathrm{NC} 01624$. Il est permis de s'interroger sur le bien-fondé de cette solution. Si la requérante ne remplissait effectivement pas les conditions pour se voir délivrer un titre de séjour, les faits de l'espèce, tels que rappelés dans la décision, ne nous paraissent pas justifier une si longue durée d'examen. Autant l'absence de contrat de travail (ou d'inscription au chômage) que de revenus suffisants (qui faisaient obstacles à la délivrance des titres sollicités) sont des données facilement vérifiables.

111. Précisons sur ce point que, au terme de l'article R. 311-6 du CESEDA, le récépissé délivré dans le cadre d'une demande de régularisation présentée sur le fondement de l'article L. 313-14 du CESEDA ne donne pas le droit de travailler à son titulaire. Le choix de donner au requérant une autorisation provisoire de séjour était donc sans conséquence pour lui. 